\title{
TRANSPORT OF NON-BROWNIAN PARTICLES IN POROUS MEDIA*
}

\author{
GUIDO KAMPEL ${ }^{\dagger}$ AND GUILLERMO H. GOLDSZTEIN ${ }^{\dagger}$
}

\begin{abstract}
Fluid in porous media flows through tortuous paths. When the fluid velocities are large enough, this tortuosity and inertial effects cause suspended particles to collide with pore walls. After each collision, a particle loses momentum and needs to be accelerated again by hydrodynamic forces. As a result, the average velocity of suspended particles may be smaller than that of the fluid. In addition, if the fluid velocity field is not macroscopically homogeneous, the retardation of the particles with respect to the fluid leads to an increase of the concentration of particles in certain regions which may eventually result in the clogging of the porous medium. This effect is of importance in flows near wells where the flow has circular symmetry and thus it is not macroscopically homogeneous. In this paper, we study the physical effect described above. We first develop a mathematical model that takes into account tortuosity and inertial effects at the pore scale. This model provides us with the average particle velocities as a function of the fluid velocity. We use this function in a second model, a macroscopic scale model, to study the evolution of concentration of particles and the development of clogging when the flow has circular symmetry.
\end{abstract} eling

Key words. porous media, suspended particles, plugging, inertial effects, mathematical mod-

AMS subject classification. $76 \mathrm{~S} 05$

DOI. $10.1137 / 090780687$

1. Introduction. A porous medium is a material that contains spaces filled with fluid embedded in a solid matrix. These fluid filled spaces are called pores or voids. A porous material is said to be permeable if fluid can flow from one region of the material to another through its voids.

Soil mass is an example of porous media. The particles that hold the soil together form the solid matrix that in this case is known as the load carrying skeleton. Fines are small particles that do not form part of this skeleton and may be carried by the flow and be trapped at other locations or exit the porous medium. The sites that trap fines are usually pore constrictions or pore throats, i.e., the thinner regions of the voids. For example, if several migrating particles reach a small pore throat simultaneously, the particles may get trapped and clog the pore throat. The clogging of pore throats decreases the overall flow in the medium, which may eventually clog; i.e., the medium would no longer be permeable. The physics that determine the dynamics of fines and the clogging of porous media is complex. More detailed discussions can be found in $[3,4,5,7,9,12,13,14,18,19,21,20,23]$. We note that fluids with fines are sometimes referred to as suspensions.

In this paper, as explained next, we study only a partial set of effects that may lead to the clogging of porous media. Fluid in porous media flows through tortuous paths, i.e., paths that are not straight. When the fluid velocities are large enough, this tortuosity and inertial effects cause fines to collide with pore walls. After each collision, a particle loses momentum and needs to be accelerated again by the fluid through hydrodynamic forces. As a result, the average velocity of fines may be smaller

${ }^{*}$ Received by the editors December 21, 2009; accepted for publication (in revised form) February 20, 2011; published electronically May 26, 2011. This research was supported by the NSF.

http://www.siam.org/journals/siap/71-3/78068.html

†School of Mathematics, Georgia Institute of Technology, Atlanta, GA 30332-0160 (kampel@ gmail.com, ggold@math.gatech.edu). 
than that of the fluid.

To simplify the discussion that will follow, we now introduce some terminology and notation. We assume that there are two length scales. One of them is the microscopic or pore scale. The second one is the macroscopic scale, which is much larger than the pore scale.

We denote by $\mathbf{v}_{p}$ and $\mathbf{v}$ what we call the microscopic and macroscopic fluid velocities, respectively. If $\mathbf{x}$ is a point in the void or pore space, $\mathbf{v}_{p}(\mathbf{x}, t)$ is the fluid velocity at $\mathbf{x}$ at time $t$. On the other hand, $\mathbf{v}$ is the average of the fluid velocity in regions much larger than the pore scale but much smaller than the macroscopic scale. More precisely, let $\Omega_{p}$ be the region in space occupied by the voids, and let $B_{\varepsilon}(\mathbf{x})$ be the ball of radius $\varepsilon$ centered at $\mathbf{x}$ (i.e., $B_{\varepsilon}(\mathbf{x})$ is the set of points whose distance to $\mathbf{x}$ is less than $\varepsilon$ ). Then, if $\varepsilon$ is much larger than the pore scale and much smaller than the macroscopic scale,

$$
\mathbf{v}=\mathbf{v}(\mathbf{x}, t) \approx \frac{1}{\left|\Omega_{p} \cap B_{\varepsilon}(\mathbf{x})\right|} \int_{\Omega_{p} \cap B_{\varepsilon}(\mathbf{x})} \mathbf{v}_{p}(\mathbf{s}, t) d \mathbf{s},
$$

where we have used the notation $|A|$ to denote the volume of any set $A$.

We denote by $\mathbf{u}$ the macroscopic fines velocity; i.e., $\mathbf{u}$ is the average of the velocities of the fines in regions much larger than the pore scale and much smaller than the macroscopic scale. If the macroscopic fluid velocity is spatially homogeneous, i.e., $\mathbf{v}$ is independent of $\mathbf{x}$, the macroscopic fines velocity $\mathbf{u}$ will also be independent of $\mathbf{x}$. Thus, the location of the maximum concentration of fines in space will travel at velocity $\mathbf{u}$, but the concentration value will not change. On the other hand, if $\mathbf{v}$ is not independent of $\mathbf{x}, \mathbf{u}$ will not be independent of $\mathbf{x}$ either. As a consequence, and as we will see in more detail later in this paper, both the location and the value of the maximum concentration of fines in space will change with time. In particular, the concentration of fines may eventually exceed some critical value that leads to the clogging of pore throats and eventually the clogging of the medium. Motivated by the clogging that is sometimes observed in petroleum and water wells, we will consider two-dimensional macroscopic flows with circular symmetry. Clearly, the macroscopic fluid velocity is not spatially homogeneous in this case; it decays with the distance to the well.

In section 2, we introduce a pore scale mathematical model that allows us to obtain the macroscopic fines velocity $\mathbf{u}$ as a function of the macroscopic fluid velocity $\mathbf{v}$. This pore scale model results from assuming that any given particle follows a tortuous path that consists of a sequence of straight channels of length $L$ and that, each time a particle reaches the end of a channel, it collides with the pore wall and loses all its momentum. Thus, each time the particle enters a new channel, it starts traveling through the channel with an initial velocity equal to zero, and it accelerates due to hydrodynamic forces.

In section 3, we introduce a macroscopic scale mathematical model that describes the time evolution of the concentration of fines. This model results from the fact that the concentration of fines is convected with velocity $\mathbf{u}$. We also propose that regions of the medium clog if and when the concentrations of fines in those regions exceed a certain critical value.

Our studies are also motivated by a series of laboratory experiments conducted by Valdes and Santamarina [23]. In fact, they propose that the physical effects we model in this paper are important factors in the plugging of porous media around wells. They used a circular sheet of emery sandpaper as a two-dimensional porous 

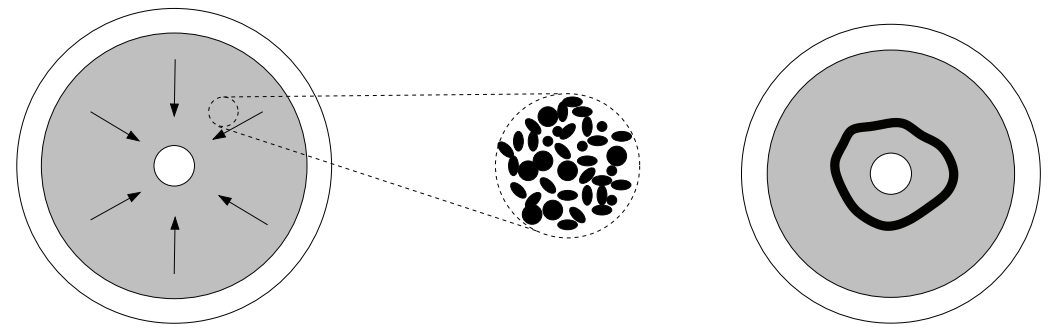

FIG. 1.1. Illustration of the experiment of Valdes and Santamarina [23]. The gray area is the two-dimensional porous medium. The arrows in the left figure indicate the average direction of the flow. The middle figure shows a microscopic look at the material, the dark region is the solid matrix, and suspension can flow only through the white region. The right figure shows a clogged medium. The region clogged appears dark.

medium. A heavy plane plate was placed on top of the rough side of the sandpaper, which rested over a foam layer on a plane surface. (The purpose of the foam is to maximize the contact between the plate and the sandpaper and to distribute this contact evenly.) The center of the sandpaper had a circular orifice. The outer edge of the porous medium was placed in contact with a suspension reservoir (fluid with fines). An imposed pressure at the outer edge, higher than the pressure at the orifice, created a suspension flow in the space enclosed between the sandpaper and the plate. The suspension flowed from the outer edge toward the orifice in the center of the sandpaper, where it exited the device (see Figure 1.1).

Both clogging and not clogging were observed in [23]. Whether clogging occurs or not depends on the parameter regime of the experiment (i.e., particle size and concentration, applied pressure, etc.). Whenever the medium clogged, it was always observed that the pore throats clogged were not evenly distributed throughout the medium. Instead, they were concentrated at a certain distance from the orifice, forming an easily observable ring of trapped particles (see Figure 1.1).

Our approach can be considered to be multiscale. As a first step we develop a pore scale model whose result is used as input in our second and final model, which belongs to the class of macroscopic models. More precisely, we keep track of the concentration of particles at the macroscopic scale. While there exist a large number of macroscopic mathematical models of migration of fines and clogging-some of the most popular include $[24,15,10,6,8,5,22,16,17,11,1]$ - our model is truly novel. Our modeling efforts focus on physical effects that, to the best of our knowledge, have not been modeled before. Our goal is to develop a simple model that captures the main features of the physical effects previously described that, in our opinion, may play a fundamental role in the clogging of porous media near wells. We believe that we accomplish our goal in the present work and that this paper will serve as a step toward more comprehensive modeling of clogging of porous media and will also suggest experiments to test hypotheses to eventually provide a better understanding of the physics involved in this complex but technologically important process.

We do want to stress that the model in the present paper should be regarded as a toy model. By this we mean a simplified model that captures the physical effects that we want to study in the simplest context possible. The advantage to studying such simple toy models is that they are easier to analyze and the results are easier to interpret. The price paid in this drastic simplification is that other physical effects that may be important are neglected. For example, in our case, in our microscopic 
model, we assume the particles to be much smaller than the size of the voids, and this is clearly not true (particularly when clogging occurs and the void space becomes even smaller). However, given the complexity of the physics involved in particle migration and clogging, we believe that the present study, as simplified as it is, has value and may serve as a building block of future more comprehensive models.

The rest of this paper is organized as follows. As previously mentioned, in section 2 we develop our pore scale model whose result is used as input in our macroscopic scale model, described in section 3 . In section 4 we explain some qualitative features of our model. In section 5 we introduce our criterion for determining which regions of the medium clog and when. In section 6 we study the behavior of our model, and in section 7 we conclude with some discussions.

\section{Macroscopic fines velocity as a function of the macroscopic fluid velocity.}

2.1. Review. Drag force on a spherical particle immersed in a fluid. Assume that an incompressible spherical particle with radius $r_{p}$ is immersed in an incompressible Newtonian fluid that extends to infinity. Assume also that the particle moves with constant velocity $\mathbf{u}$ and that the velocity of the fluid tends to the constant value $\mathbf{v}$ far away from the particle. It is well known (see [2]) that the force the fluid exerts on the particle is

$$
\mathbf{F}=6 \pi r_{p} \mu(\mathbf{v}-\mathbf{u})
$$

where $\mu$ is the fluid viscosity.

2.2. Average velocity of a particle flowing through a straight channel when the particle is initially at rest at one end of the channel. Consider a straight channel of length $L$ filled with an incompressible fluid; see Figure 2.1. Assume that the fluid within the channel flows from left to right at a constant speed $v^{\star}$. Note that, as an approximation, we assume that the fluid velocity is constant through the channel and thus does not satisfy the nonslip boundary conditions at the channel walls.

If at time $t=0$ we place a particle at the left end of the channel, it will move toward the right end following Newton's law. More precisely, if $x(t)$ is the distance between the particle and the left end of the channel at time $t$, Newton's law implies

$$
\frac{4}{3} \pi \rho_{p} r_{p}^{3} \ddot{x}=F \quad \text { and } \quad x(0)=\dot{x}(0)=0
$$

where $F$ is the force the particle experiences in the direction of the channel, $\rho_{p}$ and $r_{p}$ are the density and radius of the particle, respectively, and $\dot{x}$ and $\ddot{x}$ are the first and second the derivatives of $x$ with respect to $t$. Note that we neglect any force that the particle may experience in directions perpendicular to the channel.

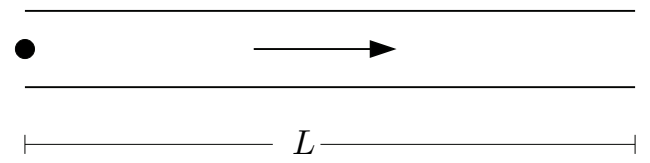

FIG. 2.1. Channel of length $L$ filled with an incompressible fluid. The arrow indicates the direction of the flow. The black circle is a particle that will move toward the right end due to hydrodynamic forces. 
Keeping only hydrodynamic forces (we neglect gravity) and approximating $F$ by the force that the particle would experience if the channel width were much larger than the particle diameter, i.e., (2.1), we have $F=6 \pi r_{p} \mu\left(v^{\star}-\dot{x}\right)$ and thus

$$
\frac{4}{3} \pi \rho_{p} r_{p}^{3} \ddot{x}=6 \pi r_{p} \mu\left(v^{\star}-\dot{x}\right) \quad \text { and } \quad x(0)=\dot{x}(0)=0,
$$

where $\mu$ is the viscosity of the fluid.

The above initial value problem can be solved explicitly. More precisely, defining

$$
\kappa=\frac{9}{2} \frac{\mu}{\rho_{p} r_{p}^{2}}
$$

we have that

$$
x(t)=v^{\star} t-\frac{v^{\star}}{\kappa}\left(1-e^{-\kappa t}\right) .
$$

Let $T$ be the time when the particle reaches the right end. Since the length of the channel is $L$, and given (2.5), we have

$$
L=v^{\star} T-\frac{v^{\star}}{\kappa}\left(1-e^{-\kappa T}\right) .
$$

We denote by $u^{\star}$ the average speed of the particle as it travels through the channel. Our goal is to find $u^{\star}$ as a function of the fluid speed $v^{\star}$ and the parameters of the system. This relation is obtained from (2.6) once we note that $u^{\star}=L / T$ and replace $T$ by $L / u^{\star}$ in that equation to obtain, after simple manipulations,

$$
1=\frac{v^{\star}}{\kappa L}\left[\frac{\kappa L}{u^{\star}}-\left(1-e^{-\frac{\kappa L}{u^{\star}}}\right)\right] .
$$

2.3. Tortuosity of flow paths in porous media. Assume now that suspension, i.e., fluid with fines, flows within the void space of a porous medium. Let $\mathbf{x}_{f}(t)$ be the path of an element of fluid. This path will not be straight; it will be tortuous. Thus, the distance traveled by the fluid element in a time interval $\left(t_{1}, t_{2}\right)$, which is $\int_{t_{1}}^{t_{2}}\left\|\dot{\mathbf{x}}_{f}(t)\right\| d t$, where $\|\cdot\|$ denotes the Euclidean norm, will be larger than the distance from $\mathbf{x}_{f}\left(t_{1}\right)$ to $\mathbf{x}_{f}\left(t_{2}\right)$; i.e.,

$$
\frac{\int_{t_{1}}^{t_{2}}\left\|\dot{\mathbf{x}}_{f}(t)\right\| d t}{\left\|\mathbf{x}_{f}\left(t_{2}\right)-\mathbf{x}_{f}\left(t_{1}\right)\right\|}>1
$$

Without being completely rigorous, we refer to the average value of the above ratio over all fluid elements and time intervals $\left(t_{1}, t_{2}\right)$ as the tortuosity. We denote the tortuosity by $\tau$. (Note that in the literature, tortuosity is sometimes defined as the square of the tortuosity as defined here.) This concept is illustrated in Figure 2.2. If a typical path traveled by an element of fluid in a porous medium is through the segments with length $\ell_{i}(1 \leq i \leq 8)$, and the distance from the initial to the final positions of the element of fluid is $L$, then the tortuosity $\tau$ is $\tau=L^{-1}\left(\sum_{1 \leq i \leq 8} \ell_{i}\right)$.

2.4. Relationship between the microscopic speed, the average velocity, and the tortuosity. Let $\mathbf{v}$ be what we have called in the introduction the macroscopic fluid velocity (see (1.1)). Let $v^{\star}$ be the average fluid speed. Then, from the definition of tortuosity $\tau$, we have that

$$
v^{\star}=\tau v, \quad \text { where } v=\|\mathbf{v}\| .
$$




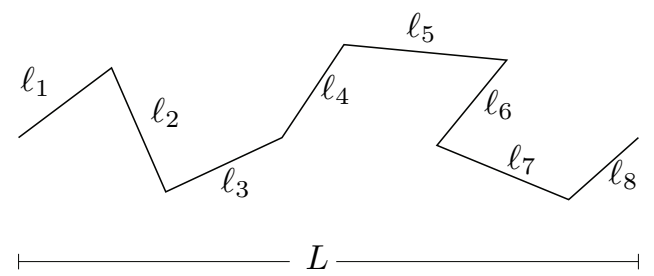

FIG. 2.2. The small segments form a typical path traveled by an element of fluid in a porous medium.

In other words, the ratio between the average of the microscopic fluid speed and the norm of the macroscopic fluid velocity is the tortuosity.

Analogously, we denote by $\mathbf{u}$ the macroscopic fines velocity - i.e., $\mathbf{u}$ is the average of the velocities of the fines in regions much larger than the pore scale but much smaller than the macroscopic scale - and by $u^{\star}$ the average speed of the fines. We also have that

$$
u^{\star}=\tau u, \quad \text { where } u=\|\mathbf{u}\| .
$$

Note that these velocities and speeds are in general functions of the spatial position $\mathbf{x}$ and time $t$; i.e., $\mathbf{v}=\mathbf{v}(\mathbf{x}, t), \mathbf{u}=\mathbf{u}(\mathbf{x}, t), v^{\star}=v^{\star}(\mathbf{x}, t)$, and $u^{\star}=u^{\star}(\mathbf{x}, t)$.

2.5. Macroscopic fines velocity as a function of the macroscopic fluid velocity. Our goal is to find $\mathbf{u}$ as a function of $\mathbf{v}$. We will assume, naturally, that $\mathbf{u}$ has the same direction as $\mathbf{v}$. Note that (2.7) provides a relationship between the speeds $u^{\star}$ and $v^{\star}$, where $L$ should be taken as a typical pore size. Thus, given (2.9) and (2.10), we have

$$
1=\frac{v}{\alpha}\left[\frac{\alpha}{u}-\left(1-e^{-\frac{\alpha}{u}}\right)\right], \quad \text { where } \alpha=\frac{\kappa L}{\tau}=\frac{9}{2} \frac{\mu L}{\tau \rho_{p} r_{p}^{2}},
$$

and as before, $v=\|\mathbf{v}\|$ and $u=\|\mathbf{u}\|$. The above equation is the key result of this section and will be used in the macroscopic model of the next section.

2.6. Behavior of the macroscopic fines velocity versus the macroscopic fluid velocity curve. Let $f(s)=s-\left(1-e^{-s}\right)$. Note that $(2.11)$ can be written as $\alpha / v=f(\alpha / u)$. From this simple observation we can draw some expected conclusions. Since $f(0)=0,0<f^{\prime}(s)<1$ for all $s>0$, and $\lim _{s \rightarrow \infty} f(s)=\infty$, then

1. $0<u<v$ for all $v>0$, and for each $v$ there exists a unique corresponding $u$;

2. $u$ is an increasing function of $v$.

The asymptotic behavior of $u$ for both small and large values of $v$ is also straightforward to obtain

$$
u \approx v \text { for } v \ll \alpha
$$

and

$$
u \approx \sqrt{\frac{\alpha v}{2}} \text { for } v \gg \alpha .
$$

A plot of $v / \alpha$ versus $u / \alpha$ is shown in Figure 2.3. 

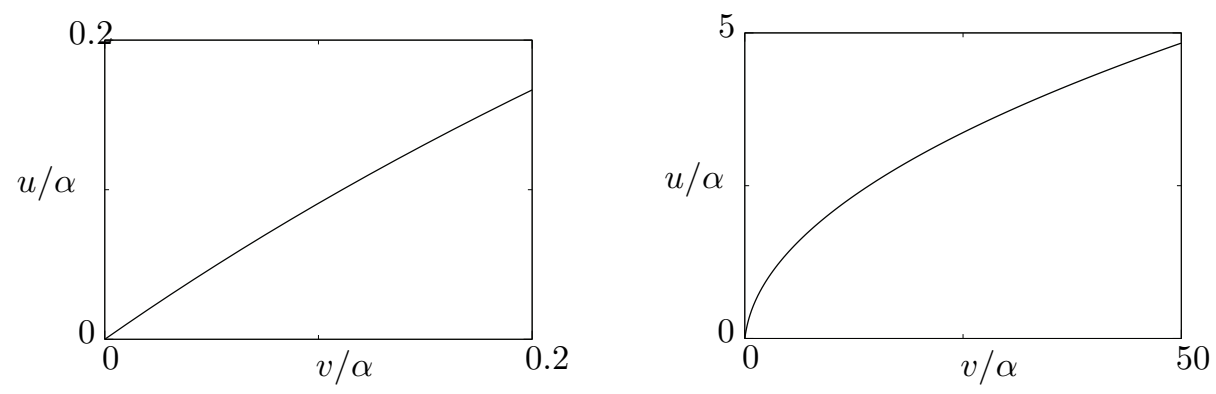

FIG. 2.3. Plot of $v / \alpha$ vs. $u / \alpha$. Both plots correspond to the same curve; they are just in different scales.

2.7. Interpretation of the parameter $\alpha$. Consider a particle initially at rest immersed in a fluid that moves at a uniform velocity away from the particle. From (2.5) we infer that $\kappa^{-1}$ is the time scale in which the particle will accelerate from rest to close to the fluid velocity.

On the other hand, if $v$ is the macroscopic speed of the fluid in a porous medium and $L$ the length of a typical pore, then the microscopic speed will be $\tau v$, and thus fluid will travel from one end of a pore to the opposite end in a period of time of order $L /(\tau v)$.

Thus, if $\kappa^{-1} \ll L /(\tau v)$, we expect that a particle traveling through a pore will have enough time to accelerate and reach the fluid velocity before the next collision, and thus we expect the macroscopic particle velocities to be similar to that of the fluid. Note that $\kappa^{-1} \ll L /(\tau v)$ is in fact the condition $v \ll \alpha$ (see (2.12)).

Now that we have identified the time scale $\kappa^{-1}$, the meaning of the parameter $\alpha$ is more clear since $\alpha=L /\left(\tau \kappa^{-1}\right)$ (see (2.11)).

\section{Evolution equation for the concentration of fines and clogging cri- teria.}

3.1. Porosity. Assume that our two-dimensional porous medium occupies the region in space $\Omega$ and that $\mathbf{x} \in \Omega$. We will denote by $\phi=\phi(\mathbf{x})$ the porosity, i.e., the local volume fraction of void or pore space. More precisely, let $B_{\delta}$ be the ball or radius $\delta$ centered at $\mathbf{x}$. Let $V_{\delta}$ be the void volume within $B_{\delta}$ (really, the void area, since we are considering two-dimensional materials). Let $\left|B_{\delta}\right|$ be the volume of $B_{\delta}$. Then, $\phi(\mathbf{x})$ is the asymptotic value of $V_{\delta} /\left|B_{\delta}\right|$ for $\delta$ much smaller than the macroscopic scale but much bigger than the pore scale:

$$
\phi(\mathbf{x})=\frac{\text { void volume in }\{\mathbf{y}:\|\mathbf{y}-\mathbf{x}\|<\delta\}}{\text { volume of }\{\mathbf{y}:\|\mathbf{y}-\mathbf{x}\|<\delta\}} .
$$

Note that $\phi=\phi(\mathbf{x})$ may depend on $\mathbf{x}$ but is independent of $t$.

3.2. Volume fraction of fines and volume fraction of fluid. We will denote by $z=z(\mathbf{x}, t)$ the volume fraction of fines. More precisely, $z(\mathbf{x}, t)$ is the asymptotic value of the volume of fines in $B_{\delta}$ divided by the volume of $B_{\delta}$ for $\delta$ much smaller than the macroscopic scale but much bigger than the pore scale:

$$
z(\mathbf{x}, t)=\frac{\text { volume of fines in }\{\mathbf{y}:\|\mathbf{y}-\mathbf{x}\|<\delta\}}{\text { volume of }\{\mathbf{y}:\|\mathbf{y}-\mathbf{x}\|<\delta\}} .
$$

Note that $z(\mathbf{x}, t)$ may depend on both $\mathbf{x}$ and $t$. Note also that the volume fraction of the fluid is $\phi(x)-z(\mathbf{x}, t)$. We will refer to $z$ as the concentration of fines. 
3.3. Fluid flow. Note also that, in the macroscopic scale, fluid is convected with what we have called the macroscopic fluid velocity $\mathbf{v}$, and the concentration of fines is convected with the macroscopic fines velocity $\mathbf{u}$. Since we assume that both particles and fluid are incompressible, the equation of mass conservation reduces to

$$
\nabla \cdot(\mathbf{v}(\phi-z)+\mathbf{u} z)=0
$$

where $\nabla \cdot$ is the divergence operator.

In the small concentration of fines limit, i.e., $z \ll \phi$, and when $\phi$ is independent of $\mathbf{x},(3.3)$ reduces to

$$
\nabla \cdot \mathbf{v}=0
$$

According to the discussions in the introduction, i.e., our motivation to study the clogging of media near wells, and given the circular geometry of wells, we assume our material to occupy the region of the plane $\left\{R_{\ell} \leq\|\mathbf{x}\|\right\}$, where $R_{\ell}$ is a constant. We will also assume that fluid flows at a known constant rate and with circular symmetry toward the inner boundary $\left\{\|\mathbf{x}\|=R_{\ell}\right\}$. Thus, introducing the radial variable

$$
r=\|\mathbf{x}\|,
$$

we have from (3.4) that the velocity $\mathbf{v}$ is of the form

$$
\mathbf{v}=-v \frac{\mathbf{x}}{r} \quad \text { with } v=\frac{A}{r}
$$

where $A$ is a known positive constant. Note that $2 \pi A$ is the rate at which fluid exits the medium through the inner boundary.

3.4. Macroscopic fines velocity as a function of $r$. The macroscopic fines velocity will also have a form similar to that of the macroscopic fluid velocity $\mathbf{v}$, i.e.,

$$
\mathbf{u}=-u \frac{\mathbf{x}}{r}
$$

where $u$ and $v$ are related by (2.11) and thus $u=u(r)$. Since we now have $v$ in terms of $r$ (see (3.6)), we can now combine (2.11) and (3.6) to obtain a relation between $u$ and $r$, namely,

$$
\frac{\alpha r}{A}=\frac{\alpha}{u}-\left(1-e^{-\frac{\alpha}{u}}\right),
$$

where $\alpha$ was defined in (2.11). Note that, from the results of subsection 2.6 and from (3.6) we have that $u$ is a decreasing function of $r$.

The asymptotic behavior of $u$ for both small and large values of $r$ is also straightforward to obtain:

$$
u \approx \frac{A}{r} \quad \text { for } r \gg \frac{A}{\alpha}
$$

and

$$
u \approx \sqrt{\frac{\alpha A}{2 r}} \text { for } r \ll \frac{A}{\alpha} .
$$

A plot of $\alpha r / A$ versus $u / \alpha$ is shown in Figure 3.1.

Note that the difference between the fluid and fines macroscopic velocities is more pronounced for small values of $r$. $v$ increases proportionally to $r^{-1}$ as $r \rightarrow 0$, but $u$ increases proportionally to $r^{-1 / 2}$.

Copyright $@$ by SIAM. Unauthorized reproduction of this article is prohibited. 

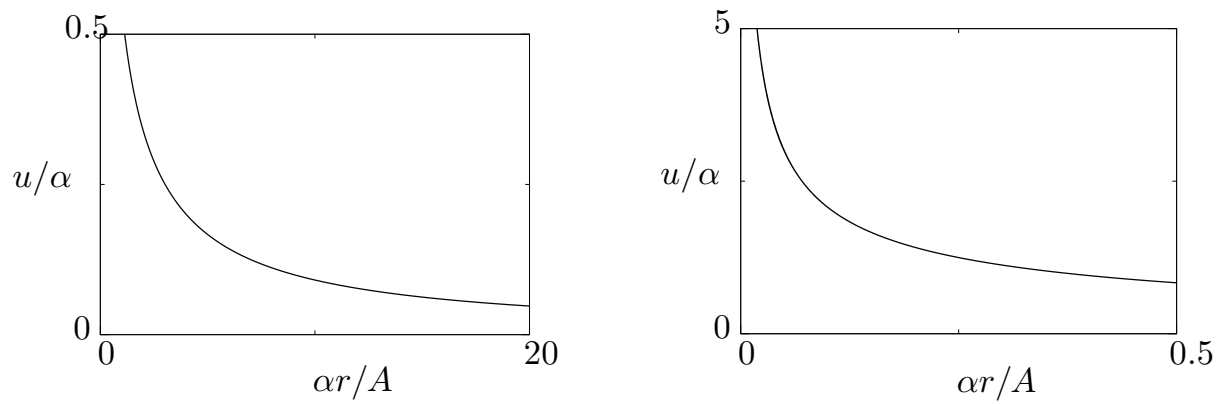

FIG. 3.1. Plot of $\alpha r / A$ vs. $u / \alpha$. Both plots correspond to the same curve; they are just in different scales.

3.5. Transport of fines. Since $z$, the concentration of fines, is convected with the macroscopic fines velocity $\mathbf{u}$, we have the following conservation equation:

$$
\frac{\partial z}{\partial t}+\nabla \cdot(\mathbf{u} z)=0
$$

Note that we neglect dispersion. Given the circular symmetry of our problem and (3.7), and assuming circular symmetry also in the initial conditions for $z$, the above equation reduces to

$$
\frac{\partial z}{\partial t}-\frac{1}{r} \frac{\partial(r u z)}{\partial r}=0
$$

where $u=u(r)$ is a function of $r$ given implicitly in (3.8).

We remark that the dependence of $u=u(r)$ on $r$ was obtained under the assumption that $z$ is small, i.e., $z \ll \phi$. Nevertheless, we will use (3.8) even when the restriction $z \ll \phi$ is not satisfied because it adds clarity to our exposition. If we do not use the assumption $z \ll \phi$, we cannot solve the equation as explicitly as we do here. Note that, while making this approximation, we keep the physical effects we are interested in modeling in this work.

Note that the evolution of the concentration of fines $z(r, t)$ is completely determined by (3.8) and (3.12) once the initial conditions are given; i.e., we need to know $z(r, 0)$.

4. Fines accumulate as they are convected toward the inner boundary. Note that the evolution equation for the concentration of fines $z$ is hyperbolic and can be solved by the method of characteristics. More precisely, we first write (3.12) as

$$
\frac{\partial z}{\partial t}-u \frac{\partial z}{\partial r}=\frac{1}{r} \frac{d(r u)}{d r} z .
$$

For convenience we define

$$
\sigma=\sigma(r)=\frac{1}{r} \frac{d(r u)}{d r} .
$$

In characteristic form, the (4.1) reads

$$
\begin{aligned}
& \dot{\bar{r}}(t)=-u(\bar{r}(t), t), \\
& \frac{d z}{d t}(\bar{r}(t), t)=\sigma(\bar{r}(t)) z(\bar{r}(t), t),
\end{aligned}
$$

Copyright (c) by SIAM. Unauthorized reproduction of this article is prohibited. 


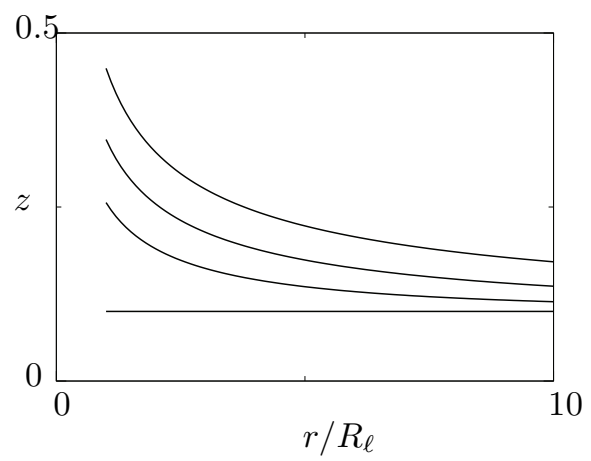

FIG. 4.1. Plot of $r / R_{\ell}$ vs. $z$ for four fixed values of $t: t=0, t=R_{\ell} / \alpha, t=5 R_{\ell} / \alpha$, and $t=100 R_{\ell} / \alpha$. We see that $z$ increases with $t$.

where $\dot{\bar{r}}$ is the derivative of $\bar{r}$ with respect to $t$.

As a first observation, we show in Appendix A.1 that $\sigma$ is always positive. Thus, the concentration of fines $z$ is always increasing along the characteristic paths; i.e., $z(\bar{r}(t), t)$ is an increasing function of time. In other words, fines accumulate as they are convected toward the inner boundary.

As a second observation, note that (4.1) is linear, and thus characteristics do not cross. In particular, we do not have formation of shocks.

In Figure 4.1 we show an example of the time evolution of $z$. We plotted $r / R_{\ell}$ versus $z$ for four fixed values of $t: t=0, t=R_{\ell} / \alpha, t=5 R_{\ell} / \alpha$, and $t=100 R_{\ell} / \alpha$. The initial condition was $z(r, 0)=0.1$. Note that $z(r, t)$ is defined only for $r \geq R_{\ell}$. The necessary calculations were computed numerically. Note that $z(r, t)$ approaches a bounded value as $t \rightarrow \infty$ for all $r$. The profile of $z$ as $t \rightarrow \infty$ is similar to the profile for $t=100 R_{\ell} / \alpha$. As expected, $z$ increases with $t$.

5. Criterion for clogging. As previewed in the introduction, our criterion for clogging is very simple. Regions of the medium clog if and when the concentrations of fines $z$ in those regions exceed a certain critical value $z_{\star}$. Note that, given the symmetry of our system, if the initial condition is homogeneous, the region that clogs first is $r=R_{\ell}$. Once this ring is clogged, there is no more flow through the medium. As we will study in later sections, clogging may or may not occur. The outcome will depend on the parameters of the system.

6. Homogeneous initial conditions. In this section we will study the evolution of concentration of fines $z$ assuming that initially the concentration of fines is homogeneous, i.e.,

$$
z(r, 0)=z_{0} \quad \text { for all } r \geq R_{\ell},
$$

where $z_{0}$ is a known positive constant.

6.1. The concentration of fines is a decreasing function of $r$. A key observation is the following

OBSERVATION 6.1. $z(r, t)$ is a decreasing function of $r$ for any fixed $t$.

Proof. Let $t$ be fixed. Let $r_{1}<r_{2}$. Let $\bar{r}_{1}(s)$ be the characteristic, i.e., a solution of the system (4.3) that satisfies $\bar{r}_{1}(t)=r_{1}$. Let $\bar{r}_{2}(s)$ be the characteristic that satisfies $\bar{r}_{2}(t)=r_{2}$. Since characteristics do not cross, we necessarily have $\bar{r}_{1}(s)<\bar{r}_{2}(s)$ for all 
$0 \leq s \leq t$. Note that (4.3) implies that

$$
z\left(r_{1}, t\right)=z_{0} e^{\int_{0}^{t} \sigma\left(\bar{r}_{1}(s)\right) d s} \quad \text { and } \quad z\left(r_{2}, t\right)=z_{0} e^{\int_{0}^{t} \sigma\left(\bar{r}_{2}(s)\right) d s} .
$$

Since, as shown in Appendix A.3, $\sigma(r)$ is a decreasing function of $r$, we conclude that $z\left(r_{1}, t\right)>z\left(r_{2}, t\right)$ for all $r_{1}<r_{2}$ and $t>0$, which concludes the proof of this observation.

6.2. Clogging may occur only at the inner boundary. Observation 6.1 implies that, if $z$ reaches the critical value $z_{\star}$, it will first reach that value at $r=R_{\ell}$. Thus, we have the following observation.

OBSERVATION 6.2. If the medium clogs, the region that clogs is the ring $r=R_{\ell}$.

6.3. Determining whether clogging occurs. Consider one fixed characteristic $\bar{r}(t)$; i.e., $\bar{r}(t)$ is a solution of system (4.3). Let $\bar{z}(t)=z(\bar{r}(t), t)$ and $\bar{u}(t)=u(\bar{r}(t))$. Note that $\bar{r}(t)$ is a strictly decreasing function of $t$, and $u(r)$ is a strictly decreasing function of $r$. Thus, $\bar{u}(t)$ is a strictly increasing function of $t$, and we can regard $t$ as a function of $\bar{u}$. Thus, $\bar{z}$ can also be regarded as a function of $\bar{u}$. In Appendix A.4 we compute $d \bar{z} / d \bar{u}$. More precisely, defining the function

$$
G(s)=\frac{1}{s}\left[\frac{1}{s}-\left(1-e^{-\frac{1}{s}}\right)\right]^{-1}\left[1-\left(1+\frac{1}{s}\right) e^{-\frac{1}{s}}\right],
$$

we show that

$$
\frac{d \bar{z}}{d \bar{u}}=\frac{1}{\alpha} G\left(\frac{\bar{u}}{\alpha}\right) \bar{z}
$$

Note that $G(s)>0$ for all $s>0$.

We now compute the value of $\bar{z}$ at the time when the characteristic $\bar{r}$ reaches the inner boundary, i.e., $\bar{r}=R_{\ell}$. This value will depend on the initial location of the characteristic, i.e., $\bar{r}_{0}=\bar{r}(0)$. Let $u_{0}$ be the macroscopic fines velocity at $r=\bar{r}_{0}$; i.e., $u_{0}$ is the solution of (3.8) when $r=\bar{r}_{0}$. Let $u_{\ell}$ be the macroscopic fines velocity at the inner boundary, $r=R_{\ell}$. Clearly $u_{\ell}>u_{0}$. We denote by $\bar{z}_{\ell}$ the value of $\bar{z}$ at the inner boundary. Given (6.4) and noticing that $\bar{z}$ should be equal to $z_{0}$ when $\bar{u}=u_{0}$ because that corresponds to the initial location of the characteristic, we have that $\bar{z}$ at the inner boundary is

$$
\bar{z}_{\ell}=z_{0} \exp \left(\int_{u_{0}}^{u_{\ell}} \frac{1}{\alpha} G\left(\frac{\bar{u}}{\alpha}\right) d \bar{u}\right)
$$

Note that

$$
\frac{d}{d s} \ln \left[1-s\left(1-e^{-\frac{1}{s}}\right)\right]=-G(s)
$$

Thus,

$$
\bar{z}_{\ell}=z_{0}\left[\frac{1-\frac{u_{0}}{\alpha}\left(1-e^{-\frac{\alpha}{u_{0}}}\right)}{1-\frac{u_{\ell}}{\alpha}\left(1-e^{-\frac{\alpha}{u_{\ell}}}\right)}\right] .
$$

Note that the further from the inner boundary the characteristic started, the larger the value of the concentration when it reached the boundary. This is clear from 

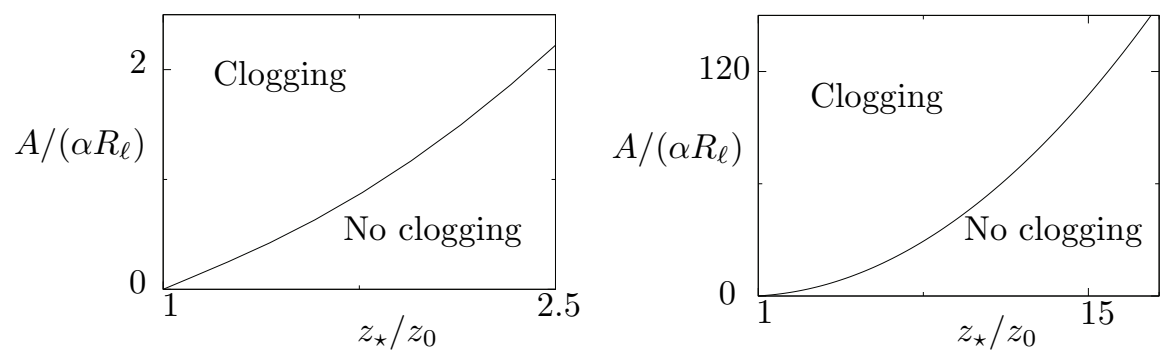

FIG. 6.1. Regions in the parameter plane $z_{\star} / z_{0}$ vs. $A /\left(\alpha R_{\ell}\right)$ where clogging does and does not occur. Both plots correspond to the same regions; they are just in different scales.

intuition and can also be seen directly in (6.5). The further from the inner boundary the characteristic started, the smaller the value of $u_{0}$ and the larger the value of $\bar{z}_{\ell}$. The limit of infinitely far corresponds to an initial macroscopic fines velocity of 0 ; i.e., $u_{0}=0$. Thus, there will be clogging if and only if $\bar{z}_{\ell}>z_{\star}$ when $u_{0}=0$.

We summarize our finding in the following observation. For convenience in the computations, we state the observation in dimensionless form. More precisely, in the below observation, $s_{\ell}=u_{\ell} / \alpha$, where $u_{\ell}$ is the macroscopic fines velocity at the inner boundary $r=R_{\ell}$ and the left-hand side of (6.9) is $\lim _{t \rightarrow \infty} z\left(R_{\ell}, t\right) / z_{0}$, the limit of the concentration of fines at $r=R_{\ell}$ divided by $z_{0}$ as $t \rightarrow \infty$ or, equivalently, the left-hand side of (6.9) is the value of $z / z_{0}$ in the limit when the characteristic that starts infinitely far reaches the inner boundary.

OBSERVATION 6.3. Let $s_{\ell}$ be the root of

$$
\frac{\alpha R_{\ell}}{A}=\frac{1}{s_{\ell}}-\left(1-e^{-\frac{1}{s_{\ell}}}\right) .
$$

The medium clogs if and only if

$$
\frac{1}{1-s_{\ell}\left(1-e^{-\frac{1}{s_{\ell}}}\right)}>\frac{z_{\star}}{z_{0}} .
$$

6.4. The parameter regime where the medium clogs. As is clear from Observation 6.3 , whether the medium clogs or not depends on the relationship between two dimensionless parameters, $A /\left(\alpha R_{\ell}\right)$ and $z_{\star} / z_{0}$. More precisely, we state Observation 6.3 in an equivalent form that is more convenient to plot in the parameter plane $z_{\star} / z_{0}$ versus $A /\left(\alpha R_{\ell}\right)$.

OBSERVATION 6.4. Let $s_{c}$ be the unique solution of

$$
\frac{1}{1-s_{c}\left(1-e^{-\frac{1}{s_{c}}}\right)}=\frac{z_{\star}}{z_{0}} .
$$

Then, the medium clogs if and only if

$$
\frac{A}{\alpha R_{\ell}} \geq \frac{s_{c}}{1-s_{c}\left(1-e^{-\frac{1}{s_{c}}}\right)} .
$$

In Figure 6.1 we display the regions in the parameter plane $z_{\star} / z_{0}$ versus $A /\left(\alpha R_{\ell}\right)$ where clogging does and does not occur. 
The parameter $z_{\star} / z_{0}$ indicates how many times the concentration of fines needs to increase for the medium to clog. Note that $A / R_{\ell}$ is the macroscopic fluid velocity $v$ at the inner boundary, and $\alpha$, defined in (2.11), is a velocity that depends on microscopic parameters of the system. Note that $\alpha$ indicates when the difference between the macroscopic fluid velocity $v$ and macroscopic fines velocity $u$ is noticeable. More precisely, $u \approx v$ if and only if $v \ll \alpha$.

The asymptotic form of the curve in Figure 6.1, which divides the parameter regions where the medium does and does not clog, can be easily obtained when $\left(z_{\star} / z_{0}-1\right) \ll 1$ and $z_{\star} / z_{0} \gg 1$. These calculations are done in Appendix A.5. Here we summarize our findings in the following observation.

OBSERVATION 6.5 . The boundary in the parameter plane $z_{\star} / z_{0}$ versus $A /\left(\alpha R_{\ell}\right)$ between the regions where the medium clogs and does not clog satisfies the following asymptotic behaviors:

$$
\frac{A}{\alpha R_{\ell}} \approx \frac{z_{\star}}{z_{0}}-1 \quad \text { if } \frac{z_{\star}}{z_{0}}-1 \ll 1
$$

and

$$
\frac{A}{\alpha R_{\ell}} \approx \frac{1}{2}\left(\frac{z_{\star}}{z_{0}}\right)^{2} \quad \text { if } \frac{z_{\star}}{z_{0}} \gg 1 .
$$

6.5. Avoiding clogging. Our work is motivated by the clogging of media around wells that is sometimes observed. Once this occurs, no more petroleum or water can be extracted from the well. Clearly, this is an undesirable effect. On the one hand, it is tempting to increase the pumping rate to maximize gains. However, our model implies that, if this pumping rate is large enough, the medium will clog. Our analysis shows the maximum rate at which fluid can be extracted without clogging the medium. First we have to estimate the parameters $z_{\star}, z_{0}$, and $\alpha$. The maximum macroscopic fluid velocity at the well to avoid clogging is $A / R_{\ell}$, solution of the system (6.10)-(6.11). Of course, our model is the result of simplifications in the attempt to isolate physical effects that we believe have a dominant contribution in the clogging of media around wells. Thus, our work should be regarded as guidelines to motivate the development of more quantitatively precise models and the initiation of related laboratory and field experiments. It should not be regarded as a model that will match experiments quantitatively with high precision.

6.6. Clogging time when clogging does occur. We now assume that clogging does occur, and our goal is to compute the time $t=t_{c}$ that it takes the material to $\operatorname{clog}$.

Let $\bar{r}(t)$ be the characteristic where clogging occurs. Thus, $\bar{r}\left(t_{c}\right)=R_{\ell}$. We define $\bar{z}(t)=z(\bar{r}(t), t)$ and $\bar{u}(t)=u(\bar{r}(t))$. Note that $\bar{z}\left(t_{c}\right)=z_{\star}$ and $\bar{u}\left(t_{c}\right)=u_{\ell}=u\left(R_{\ell}\right)$, the macroscopic fines velocity at the inner boundary. Also note that it follows from (3.8) that $u_{\ell}$ is a solution of

$$
\frac{\alpha R_{\ell}}{A}=\frac{\alpha}{u_{\ell}}-\left(1-e^{-\frac{\alpha}{u_{\ell}}}\right) .
$$

Our first step in computing the clogging time $t_{c}$ is to obtain $u_{\ell}$ from the last equation.

Let $u_{0}=u(\bar{r}(0))$; i.e., $u_{0}$ is the macroscopic fines velocity in the characteristic $\bar{r}$ at time $t=0$. Equation (6.7) and the above observations imply that

$$
z_{\star}=z_{0}\left[\frac{1-\frac{u_{0}}{\alpha}\left(1-e^{-\frac{\alpha}{u_{0}}}\right)}{1-\frac{u_{\ell}}{\alpha}\left(1-e^{-\frac{\alpha}{u_{\ell}}}\right)}\right] .
$$




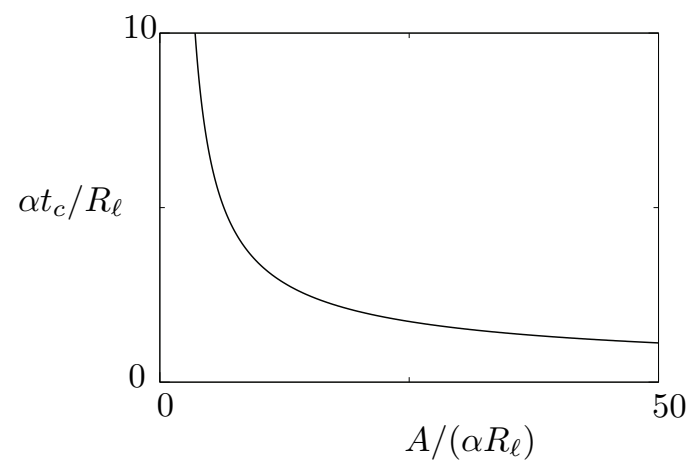

FIG. 6.2. $A /\left(\alpha R_{\ell}\right)$ vs. $\alpha t_{c} /$ Re where $z_{\star} / z_{0}=2$.

From this we can obtain $u_{0}$ after $u_{\ell}$ is obtained from (6.15).

As argued previously, we can regard $t$ as a function of $\bar{u}$. In Appendix A.6 we show that

$$
\frac{d t}{d \bar{u}}=\frac{A}{\bar{u}^{3}}\left(1-e^{-\frac{\alpha}{\bar{u}}}\right) .
$$

We can integrate the above equation, noticing that $\bar{u}=u_{0}$ when $t=0$ and $\bar{u}=u_{\ell}$ when $t=t_{c}$, to obtain

$$
\frac{\alpha^{2} t_{c}}{A}=\left(\frac{1}{2} \frac{\alpha^{2}}{u_{0}^{2}}+\left(1+\frac{\alpha}{u_{0}}\right) e^{-\frac{\alpha}{u_{0}}}\right)-\left(\frac{1}{2} \frac{\alpha^{2}}{u_{\ell}^{2}}+\left(1+\frac{\alpha}{u_{\ell}}\right) e^{-\frac{\alpha}{u_{\ell}}}\right) .
$$

Once $u_{0}$ are $u_{\ell}$ are obtained from (6.15) and (6.16), $t_{c}$ can be obtained from (6.17).

In Figure 6.2 we show a plot of $A /\left(\alpha R_{\ell}\right)$ versus $\alpha t_{c} / R_{\ell}$. As expected, the clogging time $t_{c}$ decreases with the flow rate $2 \pi A$. In particular, we show in Appendix A.7 that the clogging time $t_{c}$ decays like the $-1 / 2$ power of the flow rate. More precisely, we find that

$$
\frac{\alpha t_{c}}{R_{\ell}}=\frac{\sqrt{8}}{3}\left[\left(\frac{z_{\star}}{z_{0}}\right)^{3}-1\right] \sqrt{\frac{\alpha R_{\ell}}{A}} \quad \text { if } \frac{A}{\alpha R_{\ell}} \gg 1 .
$$

6.7. Volume of fluid extracted before the medium clogs. If clogging does occur, a quantity of interest is the volume (area in our two-dimensional problem) of fluid $V_{f}$ that exits the medium through the inner boundary before clogging occurs. In the case of wells, this is the volume of fluid extracted before the medium clogs and the well is no longer of use. Note that, if the medium clogs at $t=t_{c}$, the volume of fluid $V_{f}$ that exits the medium through the inner boundary before clogging occurs is

$$
V_{f}=2 \pi A t_{c} .
$$

In Figure 6.3 we show a plot of $A /\left(\alpha R_{\ell}\right)$ versus $V_{f} / R_{\ell}^{2}$.

The clogging time $t_{c}$ becomes unbounded as we approach from above the critical flow rate at which there is no more clogging for smaller rates. Thus, as expected, the volume extracted also becomes unbounded in this limit. On the other hand, from (6.18) we have that

$$
\frac{V_{f}}{R_{\ell}^{2}}=\frac{\sqrt{8}}{3}\left[\left(\frac{z_{\star}}{z_{0}}\right)^{3}-1\right] \sqrt{\frac{A}{\alpha R_{\ell}}} \quad \text { if } \frac{A}{\alpha R_{\ell}} \gg 1 .
$$




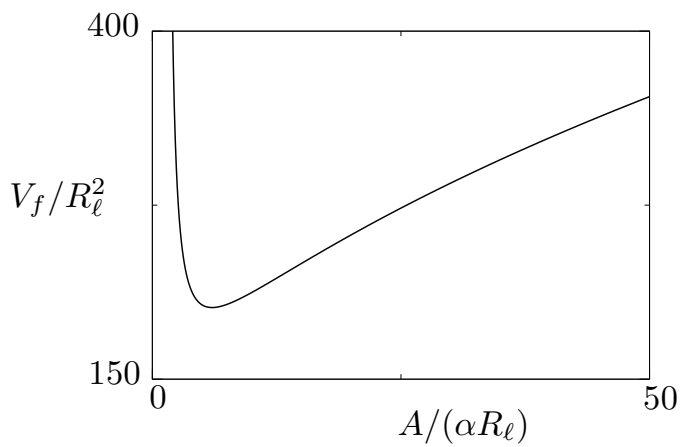

FIG. 6.3. $A /\left(\alpha R_{\ell}\right)$ vs. $V_{f} / R_{\ell}^{2}$ where $z_{\star} / z_{0}=2$.

Thus, even though the clogging time $t_{c}$ tends to 0 as the flow rate $2 \pi A$ increases, $V_{f}$ does not. In fact, $V_{f} \rightarrow \infty$ in this limit, because $t_{c}$ decreases like a $-1 / 2$ power of $A$, and thus $V_{f}=2 \pi A t_{c}$ increases like a $1 / 2$ power of $A$. As illustrated in Figure 6.3, $V_{f}$ has a unique local minimum that is also the global minimum that occurs at some finite value of $A$.

7. Conclusions. In this paper, we have introduced a multiscale model that captures and isolates the role of inertia of the particles and tortuousity of the fluid paths in the clogging of porous media near wells, where the macroscopic fluid velocity is nonhomogeneous. We refer to our model as a multiscale model because it resulted from first developing a model at the pore scale and then using the result of this model as the input of a macroscale model. While simple, the model obtained captures the physics we aimed to study. Our model has proved amenable to analysis and computations. In particular, we were able to identify dimensionless parameters that, according to our model, determine whether the medium clogs. We were also able to make detailed parameter studies. We believe that this paper will serve as a step toward more comprehensive modeling of clogging of porous media and will serve to guide laboratory and field experiments to shed more light into the physics involved in the complex and important phenomenon of clogging of porous media.

\section{Appendix.}

A.1. Calculation of $\boldsymbol{\sigma}$ as a function of $\boldsymbol{u}$. From (3.8), using the inverse function theorem and simple manipulations, we obtain

$$
\frac{d u}{d r}=-\frac{u^{2}}{A}\left(1-e^{-\frac{\alpha}{u}}\right)^{-1} .
$$

On the other hand, multiplying (3.8) by $A u / \alpha$, we get

$$
r u=A-A \frac{u}{\alpha}\left(1-e^{-\frac{\alpha}{u}}\right) .
$$

Taking the derivative with respect to $r$ in the above equation and using (A.1) and (A.2), after some manipulation we obtain that

$$
\sigma=\frac{1}{r} \frac{d(r u)}{d r}=\frac{u^{2}}{A}\left[1-\left(1+\frac{\alpha}{u}\right) e^{-\frac{\alpha}{u}}\right]\left(1-e^{-\frac{\alpha}{u}}\right)^{-1}\left[\frac{\alpha}{u}-\left(1-e^{-\frac{\alpha}{u}}\right)\right]^{-1} .
$$


Note that, for all $s>0,1+s<e^{s}$. This implies that the factor $1-(1+\alpha / u) e^{-\alpha / u}$ on the right-hand side of the above equation is positive. It is easy to show that the other factors are also positive. Thus, $\sigma(r)$ is positive for all $r$.

A.2. $\boldsymbol{\sigma}$ is an increasing function of $\boldsymbol{u}$. It is relatively easy to show that the following functions are increasing functions of $u$ :

$$
\begin{gathered}
f_{1}(u)=\frac{u^{2}}{A}\left[1-\left(1+\frac{\alpha}{u}\right) e^{-\frac{\alpha}{u}}\right], \\
f_{2}(u)=\left(1-e^{-\frac{\alpha}{u}}\right)^{-1},
\end{gathered}
$$

and

$$
f_{3}(u)=\left[\frac{\alpha}{u}-\left(1-e^{-\frac{\alpha}{u}}\right)\right]^{-1} .
$$

More precisely, taking derivatives and some simple algebraic manipulations, we have

$$
\begin{gathered}
f_{1}^{\prime}(u)=\frac{2 u}{A}\left[1-\left(1+\frac{\alpha}{u}+\frac{\alpha^{2}}{2 u^{2}}\right) e^{-\frac{\alpha}{u}}\right], \\
f_{2}^{\prime}(u)=\frac{\alpha}{u^{2}} e^{-\frac{\alpha}{u}}\left(1-e^{-\frac{\alpha}{u}}\right)^{-2},
\end{gathered}
$$

and

$$
f_{3}^{\prime}(u)=\frac{\alpha}{u^{2}}\left(1-e^{-\frac{\alpha}{u}}\right)\left[\frac{\alpha}{u}-\left(1-e^{-\frac{\alpha}{u}}\right)\right]^{-2} .
$$

It is immediate that $f_{2}^{\prime}(u)$ and $f_{3}^{\prime}(u)$ are positive for $u>0$. Noting that $1+\alpha / u+$ $\alpha^{2} /\left(2 u^{2}\right)<e^{\alpha / u}$ for $u>0$, it also follows that $f_{1}^{\prime}(u)$ is positive for $u>0$. Since $\sigma(u)=f_{1}(u) f_{2}(u) f_{3}(u)$, we conclude that $\sigma$ is an increasing function of $u$.

A.3. $\boldsymbol{\sigma}$ is a decreasing function of $\boldsymbol{r}$. From the last subsection we know that $\sigma$ is an increasing function of $u$. From the observations in subsection 3.4, we also know that $u$ is a decreasing function of $r$. Thus, we conclude that $\sigma$ is a decreasing function of $r$.

A.4. Calculation of $\boldsymbol{d} \bar{z} / \boldsymbol{d} \overline{\boldsymbol{u}}$ used in section 6.3. The variables $\bar{z}, \bar{r}$, and $\bar{u}$ are defined in subsection 6.3. Applying the implicit function theorem, we have

$$
\frac{d \bar{z}}{d \bar{u}}=\frac{d \bar{z}}{d t}\left(\frac{d \bar{r}}{d t}\right)^{-1}\left(\frac{d \bar{u}}{d \bar{r}}\right)^{-1} .
$$

The different derivatives in the above equation result from (4.3) and (A.1):

$$
\begin{aligned}
& \frac{d \bar{z}}{d t}=\sigma \bar{z}, \\
& \frac{d \bar{r}}{d t}=-\bar{u}
\end{aligned}
$$

and

$$
\frac{d \bar{u}}{d \bar{r}}=-\frac{u^{2}}{A}\left(1-e^{-\frac{\alpha}{u}}\right)^{-1} .
$$

Using the expression for $\sigma$ as a function of $u$ given by (A.3), we obtain (6.4).

Copyright $@$ by SIAM. Unauthorized reproduction of this article is prohibited. 
A.5. Asymptotic form of the curve in Figure 6.1. The curve in Figure 6.1 represents the pairs of parameters $\left(z_{\star} / z_{0}, A /\left(\alpha R_{\ell}\right)\right)$ for which there exist $s_{c}$ such that

$$
\frac{A}{\alpha R_{\ell}}=\frac{s_{c}}{1-s_{c}\left(1-e^{-\frac{1}{s_{c}}}\right)}
$$

and

$$
\frac{z_{\star}}{z_{0}}=\frac{1}{1-s_{c}\left(1-e^{-\frac{1}{s_{c}}}\right)} .
$$

Relatively straightforward calculations show that

$$
\frac{z_{\star}}{z_{0}}-1 \approx s_{c} \text { and } \frac{A}{\alpha R_{\ell}} \approx s_{c} \text { for } s_{c} \ll 1
$$

and

$$
\frac{z_{\star}}{z_{0}} \approx 2 s_{c} \text { and } \frac{A}{\alpha R_{\ell}} \approx 2 s_{c}^{2} \text { for } s_{c} \gg 1
$$

from which the validity of Observation 6.5 follows.

A.6. Calculation of $\boldsymbol{d} \overline{\boldsymbol{t}} / \boldsymbol{d} \overline{\boldsymbol{u}}$ used in section 6.6. The variables $\bar{z}, \bar{r}$, and $\bar{u}$ are defined in subsection 6.3. Applying the implicit function theorem, we have

$$
\frac{d \bar{t}}{d \bar{u}}=\left(\frac{d \bar{r}}{d t}\right)^{-1}\left(\frac{d \bar{u}}{d \bar{r}}\right)^{-1}
$$

Equation (6.16) follows from the above equation and from (A.12) and (A.13).

A.7. Asymptotic form of clogging time $\boldsymbol{t}_{\boldsymbol{c}}$ for large flows. We study the parameter regime $A /\left(\alpha R_{\ell}\right) \gg 1$. From (6.14) and some manipulation we obtain that

$$
\frac{u_{\ell}}{\alpha} \approx \sqrt{\frac{A}{2 \alpha R_{\ell}}} .
$$

Then, from (6.15) and some manipulation we obtain that

$$
\frac{u_{0}}{\alpha} \approx \frac{z_{0}}{z_{\star}} \sqrt{\frac{A}{2 \alpha R_{\ell}}} .
$$

Plugging the last two expressions into (6.17) and some manipulation leads to (6.18).

\section{REFERENCES}

[1] R. B. Bai And C. Tien, Effect of deposition in deep-bed filtration: Determination and search of rate parameters, J. Colloid Interface Sci., 231 (2000), pp. 299-311.

[2] G. K. Batchelor, An Introduction to Fluid Dynamics, Cambridge Mathematical Library, Cambridge, UK, 2000.

[3] Y. Bigno, M. B. Oyeneyin, And J. M. Peden, Investigation of pore-blocking mechanism in gravel packs in the management and control of fines migration, in Proceedings of the SPE International Symposium for Damage Control, Society of Petroleum Engineers, 1994, pp. $29-40$.

Copyright $@$ by SIAM. Unauthorized reproduction of this article is prohibited. 
[4] A. Bounroum And F. Civan, Study of particulates migration in gravel pack, in Proceedings of the SPE International Symposium for Damage Control, Society of Petroleum Engineers, 1994, pp. 75-91.

[5] C. Gruesbeck and R. E. Collins, Entrainment and deposition of fine particles in porous media, SPE J., 22 (1982), pp. 847-856.

[6] J. P. Herzig, D. M. LeClerc, AND P. L. GofF, Flow of suspensions through porous mediaApplication to deep filtration, Indust. Eng. Chem., 62 (1970), pp. 8-35.

[7] C. R. Ison and K. J. Ives, Removal mechanisms in deep bed filtration, Chem. Eng. Sci., 24 (1969), pp. 717-729.

[8] P. R. Johnson, N. Sung, And M. Elimelech, Colloid transport in geochemically heterogeneous porous media: Modeling and measurements, Environ. Sci. Technol., 30 (1996), pp. 32843293.

[9] K. C. Khilar and H. S. Fogler, Migration of Fines in Porous Media, Kluwer Academic Publishers, Dordrecht, The Netherlands, 1998.

[10] B. E. Logan, D. G. Jewett, R. G. Arnold, E. J. Bouwer, and C. R. O'Melia, Clarification of clean-bed filtration models, J. Environ. Eng., 121 (1995), pp. 869-873.

[11] J. D. Logan, Transport Modeling in Hydrogeochemical Systems, Springer-Verlag, New York, 2001.

[12] T. W. Muecke, Formation fines and factors controlling their movement in porous media, J. Petroleum Tech., 31 (1979), pp. 144-150.

[13] M. B. Oyeneyin, J. M. Peden, A. Hosseini, And G. Ren, Factors to consider in the effective management and control of fines migration in high permeability sands, in Proceedings of the SPE European Formation Damage Conference (The Hague, The Netherlands, 1995), 1995, pp. 355-360.

[14] V. B. Pandya, S. Bhuniya, and K. C. Khilar, Existence of a critical particle concentration in plugging of a packed bed, AIChE J., 44 (1998), pp. 978-981.

[15] R. Rajagopalan and C. Tien, Trajectory analysis of deep bed filtration using the sphere in cell porous media model, AIChE J., 22 (1976), pp. 523-533.

[16] M. Sahimi, G. R. Gavalas, And T. T. Tsotsis, Statistical and continuum models of fluid-solid reactions in porous media, Chem. Eng. Sci., 45 (1990), pp. 1443-1502.

[17] J. E. Saiers, G. M. Hornberger, And L. Liang, First- and second-order kinetics approaches for modeling the transport of colloidal particles in porous media, Water Resour. Res., 30 (1994), pp. 2499-2506.

[18] R. Sakthivadivel And H. A. Einstein, Clogging of porous column of spheres by sediment, ASCE J. Hydraulic Engrg., 96 (1970), pp. 461-472.

[19] M. M. Sharma And Y. C. Yortsos, Fines migration in porous media, AIChE J., 33 (1987), pp. $1654-1662$.

[20] J. L. Sherard, L. P. Dunnigan, and J. R. Talbot, Basic properties of sand and gravel filters, J. Geotech. Engrg., 110 (1984), pp. 684-701.

[21] J. L. Sherard, L. P. Dunnigan, and J. R. Talbot, Filters for silts and clays, J. Geotech. Engrg., 110 (1984), pp. 701-718.

[22] H. Soo And C. J. RADKE, A filtration model for the flow of dilute, stable emulsions in porous media -i. Theory, Chem. Eng. Sci., 41 (1986), pp. 263-272.

[23] J. R. Valdes and J. C. Santamarina, Particle clogging in radial flow: Microscale mechanisms, SPE J., 11 (2006), pp. 193-198.

[24] K. Yao, M. T. Habibian, and C. R. OMelia, Water and waste water filtration: Concepts and applications, Environ. Sci. Technol., 5 (1971), pp. 1105-1112.

Copyright $@$ by SIAM. Unauthorized reproduction of this article is prohibited. 